\title{
Biologics for the treatment of pyoderma gangrenosum in ulcerative colitis
}

\author{
K Arivarasan, Vaishali Bhardwaj, Sukrit Sud, Sanjeev Sachdeva, Amarender Singh Puri \\ Department of Gastroenterology, GB Pant Hospital, New Delhi, India
}

Pyoderma gangrenosum (PG) is an uncommon extra-intestinal manifestation of inflammatory bowel disease (IBD). Despite limited published literature, biologics have caused a paradigm shift in the management of this difficult-to-treat skin condition. The clinical data and outcomes of three patients with active ulcerative colitis and concurrent PG treated with biologics (infliximab two and adalimumab one) are reviewed in this report. Biologics were added because of the sub-optimal response of the colonic symptoms and skin lesions to parenteral hydrocortisone therapy. All three patients showed a dramatic response to the addition of the biologics. In view of the rapid healing of the skin lesions, superior response rate, and the additional benefit of improvement in the underlying colonic disease following treatment, anti-tumor necrosis factor blockers should be considered as a first line therapy in the management of PG with underlying IBD. (Intest Res 2016;14:365-368)

Key Words: Inflammatory bowel disease; Extra-intestinal manifestations; Biological products; Pyoderma gangrenosum; Colitis, ulcerative

\section{INTRODUCTION}

Pyoderma gangrenosum (PG) is an uncommon extraintestinal manifestation of IBD. It occurs in $0.4 \%$ to $2 \%$ of patients with IBD, with a slightly higher prevalence in patients with UC than in those with CD. ${ }^{1,2}$ Approximately $36 \%$ to $50 \%$ of patients with PG have underlying IBD. ${ }^{2}$ The correlation between clinical activity of the intestinal disease and the appearance of skin lesions is controversial. As this is a highly debilitating skin condition, rapid healing is the primary therapeutic objective in these patients.

The conventional treatment of PG includes local wound care, antibiotics, various systemic and topical immunomodulators, and corticosteroids. ${ }^{3,4}$ However, responses to these therapies are highly variable. With the advent of biologics, especially anti-tumor necrosis factor (anti-TNF) $\alpha$ inhibitors,

Received April 28, 2016. Revised June 4, 2016.

Accepted June 15, 2016.

Correspondence to Amarender Singh Puri, Department of Gastroenterology, GB Pant Hospital, New Delhi 110002, India. Tel: +91-1123233001, Fax: +911123234242,E-mail: amarender.puri@gmail.com

Financial support: None. Conflict of interest: None. there has been an increased interest in their role in the management of PG. Despite limited published literature, biologics have caused a paradigm shift in the management of this difficult-to-treat skin condition. ${ }^{5-7}$

Here, we report three cases of PG with UC, successfully treated using biologics. Prior to this report, there have only been two brief reports of PG treated with biological therapy, from Asia., ${ }^{8,9}$

\section{CASE REPORTS}

The clinical data of three patients with active UC and PG admitted to the gastroenterology ward of our institute between 2012 and 2016 and treated with biologics were reviewed. A total of $324 \mathrm{UC}$ patients were seen in the department of gastroenterology between 2012 and 2016. Of these, three (1\%) were diagnosed with PG. Data collected included age, duration and severity of bowel disease; the location, number, and duration of the skin lesions; prior treatment, time to response and healing of PG lesions following treatment with biologics; and status of bowel disease following treatment with biologics. The improvement of the skin le-

\footnotetext{
๑ Copyright 2016. Korean Association for the Study of Intestinal Diseases. All rights reserved.

This is an Open Access article distributed under the terms of the Creative Commons Attribution Non-Commercial License (http://creativecommons.org/licenses/by-nc/4.0)

which permits unrestricted non-commercial use, distribution, and reproduction in any medium, provided the original work is properly cited.
} 
sions was based on the decrease in the size and depth of the ulcer, and the appearance of healthy granulation tissue over the previously exposed deep fascia.

\section{Case 1}

A 40-year-old male presented with complaints of bloody diarrhea of 3 months' duration. Five days after the onset of blood in stools, he noticed small vesicular lesions over the dorsum of the right foot, followed by the appearance of similar lesions on the left foot, buttocks, and hands. These lesions subsequently formed ulcers. The size of the largest lesion was $5.5 \times 4.5 \mathrm{~cm}$. The base of the penetrating ulcer exposed the deep fascia of the tendon of his foot, with complete denudation of the skin and subcutaneous tissue (Fig. 1A).

At admission, the patient had a clinical Mayo score of 9 . He had pallor, as well as ulcers over dorsum of both feet (Fig. 1A) and over the left buttock. Laboratory evaluation revealed a hemoglobin level of $8.6 \mathrm{~g} / \mathrm{dL}$, total leukocyte count of 10,800 cells $/ \mu \mathrm{L}$, total serum protein level of $5.2 \mathrm{~g} / \mathrm{dL}$, and a serum albumin level of $2.9 \mathrm{~g} / \mathrm{dL}$. Colonoscopic evaluation revealed diffuse loss of vascular pattern, multiple superficial ulcers, and oozing of blood involving the entire length of the colon. The extent of the disease was therefore classified as E3 as per the Montreal classification, and the colonoscopic severity was 7 as per ulcerative colitis endoscopic index of severity (UCEIS). A diagnosis of UC with acute exacerbation

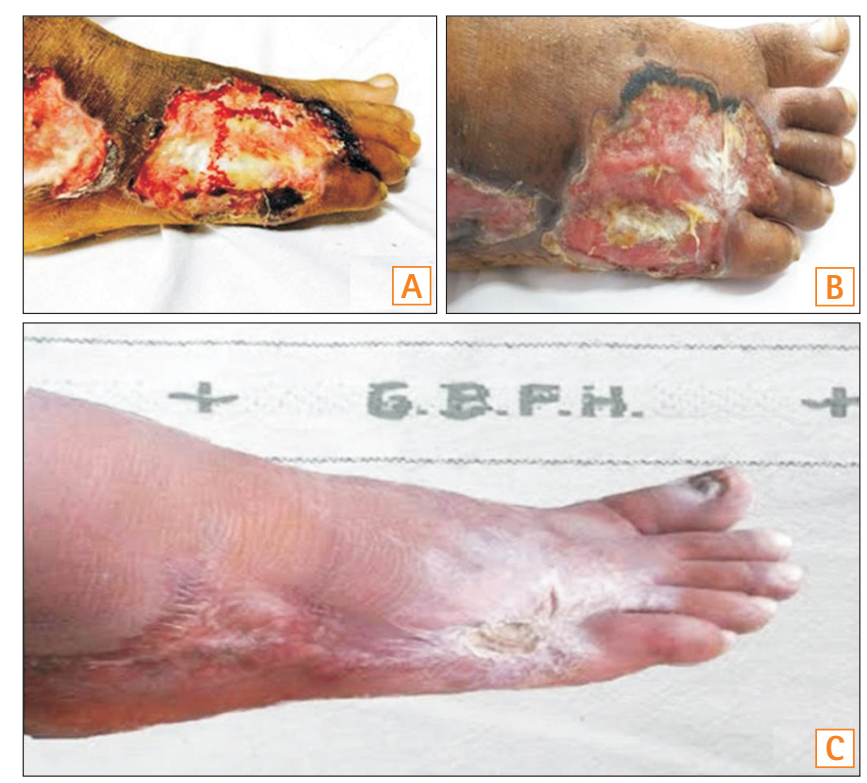

Fig. 1. Pyoderma gangrenosum involving right foot at baseline (A), 1 week after initiation of infliximab therapy (B) and 6 months posttherapy (C). and PG was made.

The patient was started on intravenous hydrocortisone (100 mg every 8 hours), mesalamine, and azathioprine. Topical antibiotics and dressing were used to treat the skin lesions. The patient showed partial improvement of bowel symptoms; however, there was no improvement in the skin lesions, even after 1 week of intensive steroid therapy. Hence, he was started on intravenous infliximab at a dose of $5 \mathrm{mg} /$ $\mathrm{kg}$. Following infliximab administration, he showed signs of healing at the peripheral margin of the skin lesions, coupled with appearance of granulation tissue over the previously visible deep fascia of the foot within 1 week of receiving the first dose (Fig. 1B). The transverse diameter of the lesion on the lateral malleolus of the right foot was decreased by approximately $1 \mathrm{~cm}$ within 1 week. Subsequently, he received the remaining two induction doses of infliximab at weeks 2 and 6 . The skin lesions continued to heal, and he was discharged with a prescription of oral mesalamine and azathioprine. After 6 months, the skin lesions had healed completely, albeit with extensive scarring on the dorsum of the foot (Fig. 1C). His bowel symptoms were also in remission at the time of the last follow-up.

\section{Case 2}

A 36-year-old male who had been diagnosed with UC 8 years prior presented with a 2-month history of loose stools mixed with blood, as well as ulcers over the left foot and the dorsum of the left thumb.

At admission, the patient had a clinical Mayo score of 8 . He had deep ulcers over the lateral malleolus of the left foot and the left thumb (Fig. 2A and C). Sigmoidoscopy was suggestive of severe colitis with a UCEIS score of 6 . The patient was treated with intravenous hydrocortisone (100 mg every 8 hours), mesalamine, azathioprine, local therapy with topical antibiotics, and steroids, and daily dressing of the wound. He showed partial improvement of bowel symptoms but no improvement of skin lesions. Hence, he was subcutaneously administered a bolus dose of $160 \mu \mathrm{g}$ adalimumab. The skin lesions showed improvement by the fourth day, with the appearance of healthy granulation tissue. Twelve weeks following adalimumab therapy, the lesions had healed completely (Fig. 2B and D). The patient was gradually weaned off steroids, and administered a second dose of $80-\mu$ g adalimumab after 2 weeks. His skin lesions had healed significantly, but he had to undergo an emergency colectomy following colonic perforation during the colonoscopy that was performed to evaluate the extent of the disease. During surgery, a $0.5 \times 0.5$ 

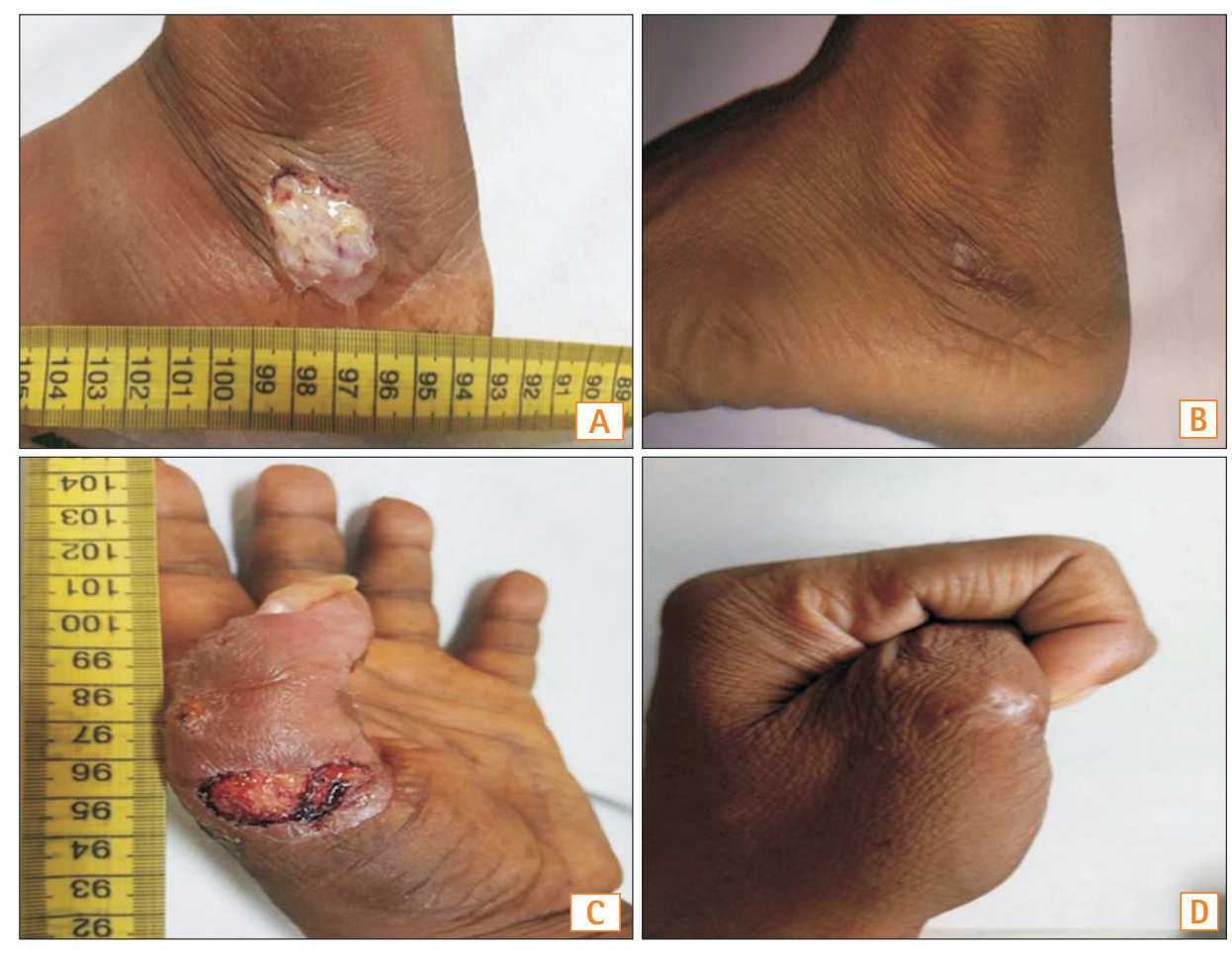

Fig. 2. Pyoderma gangrenosum involving left foot and left thumb at baseline $(A, C)$ and 12 weeks after initiation of adalimumab therapy $(B, D)$. cm perforation was detected in the sigmoid colon, with minimal peritoneal soiling. Adalimumab was discontinued after the second dose, as the skin lesions had healed considerably, and the patient had already undergone a colectomy.

\section{Case 3}

A 22-year-old male, diagnosed with UC 6 years prior with a disease extent of E3 as per the Montreal classification, presented with a 1-month history of increased frequency of stools mixed with blood. With the worsening of bowel symptoms, he also noticed a vesicular lesion on his left arm, which gradually progressed to a large ulcerative lesion. The patient had been on mesalamine and azathioprine prior to the current presentation.

At admission, he had a clinical Mayo score of 8. A deep ulcer $5 \times 3 \mathrm{~cm}$ in size was found on his left arm. Laboratory investigations revealed a hemoglobin level of $8.3 \mathrm{~g} / \mathrm{dL}$, an ESR of $55 \mathrm{~mm} / \mathrm{h}$, and a serum albumin level of $2.8 \mathrm{~g} / \mathrm{dL}$. Sigmoidoscopy was suggestive of severe colitis. A diagnosis of acute exacerbation of UC with PG was made. The patient received intravenous hydrocortisone (100 mg every 8 hours), mesalamine, and azathioprine, as well as local therapy using topical antibiotics, steroids, and daily dressing. He showed improvement in his bowel symptoms after 1 week of therapy; however, there was no improvement in skin lesions. Hence, he was started on intravenous infliximab at a dose of $5 \mathrm{mg} / \mathrm{kg}$. The second dose was administered 2 weeks later. The skin lesions showed considerable improvement, evidenced by the reduction in size and the appearance of healthy granulation tissue at the periphery after the second dose of infliximab. A third dose of infliximab was administered at 6 weeks. Steroids were tapered off, and mesalamine and azathioprine were continued. After 6 months, the patient showed complete resolution of the skin lesion (which was replaced by scar tissue), and his bowel symptoms were in complete remission.

\section{DISCUSSION}

The treatment of PG has seen a paradigm shift after the introduction of therapy directed against TNF- $\alpha$, which is a proinflammatory cytokine expressed in skin tissue of patients with PG. It contributes to the recruitment of inflammatory cells to the skin by increasing the expression of adhesion molecules in the vasculature of the inflamed tissue. ${ }^{10}$ Initially, the successful use of anti-TNF blockers such as infliximab and adalimumab in the treatment of PG was reported in anecdotal case reports. ${ }^{5-7}$ Three of the major studies on the use of biologics in PG with IBD have been tabulated in Table 1. In the only randomized controlled trial of infliximab for the treatment of $\mathrm{PG}, 13$ patients were randomized to inflix- 
Table 1. Major Published Studies on Biologics in Pyoderma Gangrenosum with IBD

\begin{tabular}{|c|c|c|c|}
\hline Author (year) & Type of study & Biologic used & Comments \\
\hline Regueiro et al..$^{6}$ (2003) & $\begin{array}{l}\text { Multicenter } \\
\text { retrospective study }\end{array}$ & Infliximab & $n=13 ;$ overall response rate to biologics, $23 \%$ \\
\hline Brooklyn et al. ${ }^{5}$ (2006) & $\begin{array}{l}\text { Randomized } \\
\text { controlled trial }\end{array}$ & Infliximab & $\begin{array}{l}\text { Infliximab }(n=13) \text {, placebo }(n=17) \text {, overall response rate to biologics } \\
\text { and placebo were } 69 \% \text { and } 21 \% \text {, respectively }\end{array}$ \\
\hline Agarwal et al.' (2013) & Systematic review & $\begin{array}{l}\text { Infliximab or } \\
\text { adalimumab or both }\end{array}$ & $\begin{array}{l}\text { Infliximab ( } n=34) \text {, adalimumab }(n=4) \text {, both infliximab and } \\
\text { adalimumab }(n=2) \text {; overall response rate of biologics, } 92 \%\end{array}$ \\
\hline
\end{tabular}

imab, and 17 were randomized to the placebo group. After 2 weeks, $46 \%$ of the patients in the infliximab group showed improvement, in comparison to only $6 \%$ in the placebo group. Complete remission of the skin lesions at week 6 was documented in $21 \%$ of the patients. ${ }^{5}$ However, even in this study, only six patients with PG had UC, of which only two were randomized to receive infliximab. Prior to this randomized controlled trial, Regueiro et al. ${ }^{6}$ treated 13 patients with IBD (CD, 12; UC, 1) and medically refractory PG with $5 \mathrm{mg} /$ $\mathrm{kg}$ infliximab. All 13 patients showed complete remission of the skin lesions, although 10 of the 13 patients required further maintenance dosing. All the patients were successfully tapered off corticosteroids completely. Though the data showing results of treatment of UC-associated PG with biological therapy is still rather limited, there is compelling evidence for the use of biologics. Even though both infliximab and adalimumab have been used successfully in the management of UC-associated PG, infliximab is more effective, making it the first choice therapy (Table 1).

In our report, all three patients showed a successful outcome despite the fact that some of the lesions of PG had penetrated to the deep fascia of the foot. Complete healing of the skin wound (albeit with scarring) was documented at 6 months after initiation of the biologic therapy. In all three patients, we used only the induction doses of the biologics, and there was no recurrence of PG after the discontinuation of the drugs.

In conclusion, in view of the rapid healing of the skin lesions, superior response rate, and the additional benefit of improvement in the underlying colonic disease following treatment with these agents, anti-TNF blockers should be considered as a first line therapy in the management of PG. The rationale for the use of biologic therapy is even stronger in the sub-group in which PG is associated with steroid refractory active colonic disease.

\section{REFERENCES}

1. Menachem Y, Gotsman I. Clinical manifestations of pyoderma gangrenosum associated with inflammatory bowel disease. Isr Med Assoc J 2004;6:88-90.

2. Rothfuss KS, Stange EF, Herrlinger KR. Extraintestinal manifestations and complications in inflammatory bowel diseases. World J Gastroenterol 2006;12:4819-4831.

3. Chow RK, Ho VC. Treatment of pyoderma gangrenosum. J Am Acad Dermatol 1996;34:1047-1060.

4. Brooklyn T, Dunnill G, Probert C. Diagnosis and treatment of pyoderma gangrenosum. BMJ 2006;333:181-184.

5. Brooklyn TN, Dunnill MG, Shetty A, et al. Infliximab for the treatment of pyoderma gangrenosum: a randomised, double blind, placebo controlled trial. Gut 2006;55:505-509.

6. Regueiro M, Valentine J, Plevy S, Fleisher MR, Lichtenstein GR. Infliximab for treatment of pyoderma gangrenosum associated with inflammatory bowel disease. Am J Gastroenterol 2003;98:1821-1826.

7. Agarwal A, Andrews JM. Systematic review: IBD-associated pyoderma gangrenosum in the biologic era, the response to therapy. Aliment Pharmacol Ther 2013;38:563-572.

8. Hayashi H, Kuwabara C, Tarumi K, Makino E, Fujimoto W. Successful treatment with infliximab for refractory pyoderma gangrenosum associated with inflammatory bowel disease. J Dermatol 2012;39:576-578.

9. Ueda M, Katoh M, Tanizaki H, Tanioka M, Matsumura Y, Miyachi Y. Refractory pyoderma gangrenosum associated with ulcerative colitis successfully treated with infliximab. Dermatol Online J 2012;18:12.

10. Bister V, Mäkitalo L, Jeskanen L, Saarialho-Kere U. Expression of MMP-9, MMP-10 and TNF-alpha and lack of epithelial MMP1 and MMP-26 characterize pyoderma gangrenosum. J Cutan Pathol 2007;34:889-898. 